\title{
Surgical management of intracranial capillary hemangiomas in children: report of 2 cases
}

\author{
Paul A. Grabb, MD \\ Department of Neurosurgery, Children's Mercy Hospital, Kansas City, Missouri; and University of Kansas School of Medicine, \\ Kansas City, Kansas \\ Two cases of intracranial capillary hemangiomas (ICHs) occurring in children are presented to highlight the surgical \\ challenges encountered with these extremely rare lesions. The author describes their clinical presentation, preoperative \\ imaging features, intraoperative findings, and operative management. The pertinent literature is reviewed. Recommen- \\ dations for preoperative planning and intraoperative management are made based on the author's experience and the \\ literature for when $\mathrm{ICH}$ is considered in the differential diagnosis and encountered intraoperatively.
}

http://thejns.org/doi/abs/10.3171/2015.7.PEDS14627

KEY WORDS intracranial capillary hemangioma; children; oncology

$\mathrm{C}$ APILLARY hemangiomas of the central nervous system are composed of a compact arrangement of capillaries lined by plump or flattened endothelial cells without intervening neural tissue, possessing histological features similar to those of lobular capillary hemangioma of the skin and capillary hemangiomas of infancy, except that intracranial capillary hemangiomas tend to be more cellular than their cutaneous counterparts. ${ }^{1}$ Intracranial capillary hemangiomas (ICHs) are distinctly different from cavernous malformations, distinguishable by their appearance on imaging, propensity for hemorrhage, and histopathology. MRI of cavernous malformations tends to display an intraparenchymal lesion with little to no enhancement, evidence of chronic prior hemorrhage, and rarely shows signal changes consistent with extensive surrounding edema. This is in contradistinction to ICHs on MRI that are often not intraparenchymal, are brightly enhancing, infrequently show evidence of prior hemorrhage, and can be associated with extensive perilesional edema. Clinically it is unusual for patients with an ICH to present because of hemorrhage, whereas hemorrhage is a common cause of clinical presentation in patients with cavernous malformations. Unlike ICHs as described above, cavernous malformations, histopathologically, have dilated capillaries with thin-walled endothelial cells and often have evidence of prior hemorrhage. ICHs are considered at the benign end of the spectrum of proliferative vascular lesions that include papillary endothelial hyperplasia, hemangioendothelioma, cellular hemangioma, hemangiopericytoma, and angiosarcoma. ${ }^{18}$ They are considered more hamartomatous than neoplastic.

ICHs come to clinical attention infrequently. Presentation falls predictably into three categories as with most intracranial masses: symptoms of raised ICP, seizure, or cranial nerve or motor deficit. Only 14 children with ICHs have been reported in the literature (Table 1). Two more pediatric cases of ICH are reported here in conjunction with a review of the literature to put forth a perioperative strategy and highlight the surgical challenges and lessons learned with these exceedingly rare intracranial lesions.

\section{Case Reports}

\section{Case 1}

A 10 -year-old boy presented with a 2- to 3-week history of worsening headache. He was an otherwise completely healthy child. There was no family history of intracranial neoplasms or vascular malformations.

\section{Examination}

His cardiac and cutaneous examinations were normal. He had mild papilledema, and no focal neurological defi- 


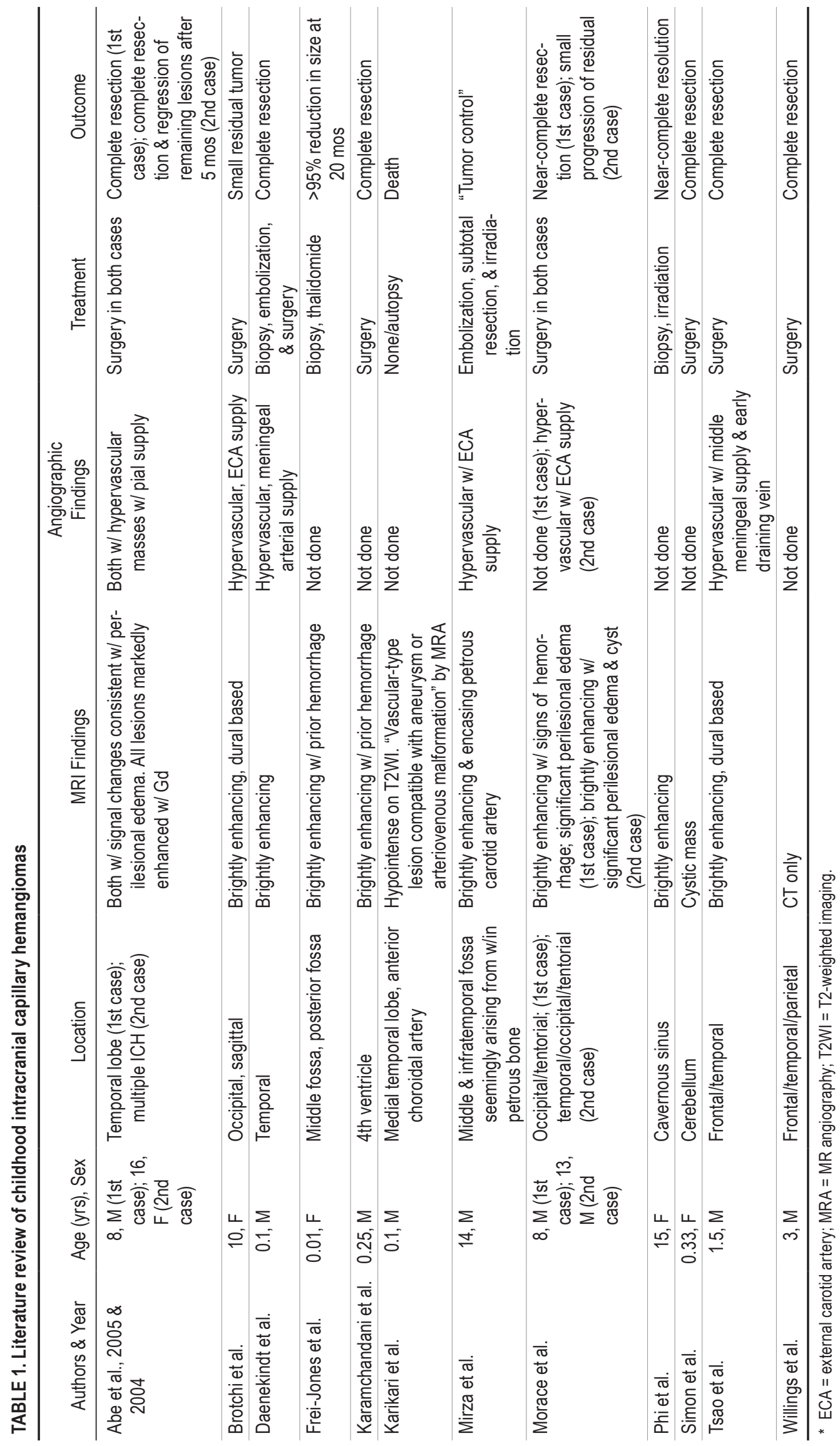


cits. His visual fields were normal. An occipital lesion based on the tentorium was diagnosed preoperatively (Fig. 1). The lesion enhanced brightly and was sharply circumscribed. There was significant perilesional edema. Flow voids, draining veins, and signs of prior hemorrhage were absent on the imaging studies.

\section{Operation}

He underwent an image-guided occipital craniotomy. Despite preoperative dexamethasone, intraoperative mannitol, mild hypocarbia, head elevation, and no pronounced neck flexion, there was profound occipital lobe swelling. The occipital lobe, however, was able to be safely elevated off the tentorium-based lesion while utilizing the intraoperative microscope. A bright red, obviously vascular mass with numerous arterial feeders and a red draining vein was encountered. Although the MRI findings were not consistent with an arteriovenous malformation, there was the concern of failure to recognize a high-flow vascular lesion preoperatively given the intraoperative appearance. It was decided to halt the case and obtain an angiogram under the same anesthetic. The dura could not be closed because of brain swelling and the bone flap was not fixated but left loose under the scalp to accommodate the occipital lobe swelling. The angiogram with both vertebral, external carotid, and internal carotid artery injections displayed only a faint blush on the vertebral injection, but no early draining vein (Fig. 2). Intravenous dexamethasone was administered between operations. The child was returned to the operating room the next day and the lesion was resected. Numerous feeders from the tentorium were coagulated and the lesion removed en bloc. The occipital swelling at this point was inconsequential and dura and bone flap were closed and fixated, respectively. No blood transfusion was necessary for either operation.

\section{Postoperative Course}

The child's neurological examination was normal. Pathological findings were diagnostic for capillary hemangio$\mathrm{ma}$, and this diagnosis was corroborated by pathological review at another academic institution (Fig. 3). Contrastenhanced cranial MRI 30 months postoperatively showed no evidence of residual or recurrent lesion (Fig. 4).

\section{Case 2}

A 14-year-old boy presented with worsening diplopia a few days following a minor bicycle accident. He was a healthy child by history. There was no family history of intracranial neoplasms or vascular malformations.

\section{Examination}

The patient's cardiac and cutaneous examinations were normal. On neurological examination he was alert and interacted appropriately. There was a left abducens nerve paresis, and mild left central facial weakness. A mass based in the region of the sylvian fissure was diagnosed preoperatively (Fig. 5). Like the imaging features in Case 1, the almost 3-cm lesion enhanced brightly and was sharply circumscribed. There was significant perilesional edema. Flow voids, draining veins, and evidence of prior hemorrhage were absent on imaging.
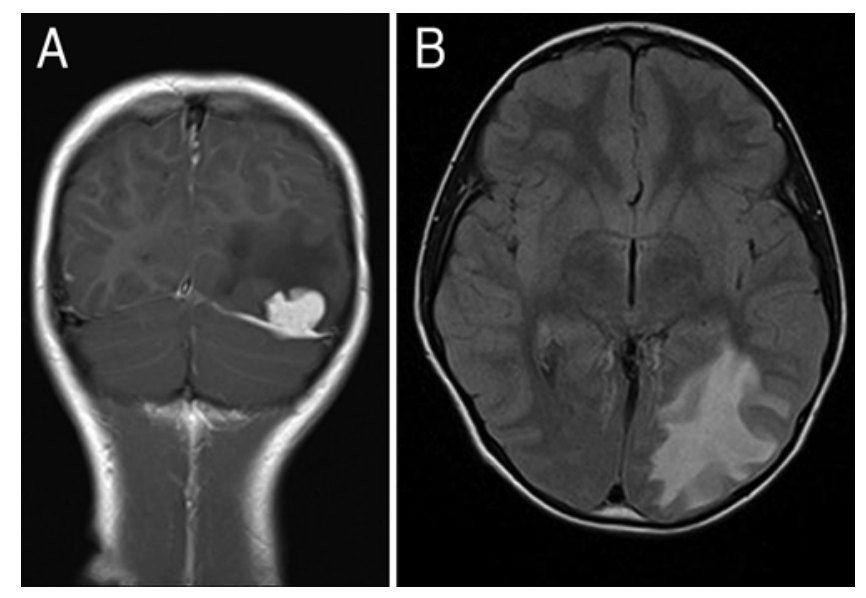

FIG. 1. Case 1. A: Coronal T1-weighted Gd-enhanced MR image showing brightly enhancing well-circumscribed mass based on the tentorium with perilesional signal change consistent with edema. B: Axial FLAIR image demonstrating left occipital signal change consistent with edema.

\section{Operation}

The patient underwent an image-guided right frontoparietal craniotomy. Again, despite preoperative dexamethasone, intraoperative mannitol, hypocarbia, neutral neck position, and head elevation, there was profound cerebral swelling. After durotomy, the mass auto-delivered itself through the sylvian fissure as the brain mushroomed up through the dural opening. Intraoperative ultrasound was used to look for an intraparenchymal hematoma, but no satisfactory images could be obtained with the probe positioned atop the mushrooming brain. Once again utilizing the intraoperative microscope, visualization of the lesion showed it to be a bright red, obviously vascular lesion similar to a varix containing oxygenated blood and numerous communications both, venous and arterial, with the sylvian vessels. Any mobilization of the lesion brought forth brisk bleeding from the sylvian fissure. Again, there was concern that there was failure to recognize a highflow vascular lesion preoperatively, and it was decided to halt the case and obtain an angiogram under the same anesthetic. Additionally, since the brain swelling was so profound, cranial CT was performed to assess for intracranial hemorrhage deep to the lesion. The craniotomy was expanded and the dura widely opened without replacement of the bone flap before closing. The CT scan revealed a hematoma medial and superior to the mass, along with the known brain swelling (Fig. 6). Internal and external carotid artery angiograms displayed no abnormal blush or early draining vein (Fig. 7). The child was immediately returned to the operating room where the lesion was resected en bloc. Numerous vessels spanned between the sylvian branches and the lesion. There was one significant arterial supply coming from a branch of the middle cerebral artery (MCA) that was either avulsed in association with the spontaneous herniation of the lesion upon dural opening or in association with microsurgical manipulation. Given the near auto-delivery of this mass upon simply opening the dura, the former etiology of spontaneous avlusion was suspected. This avulsion from a branch of the MCA was the source of the hematoma depicted on the 


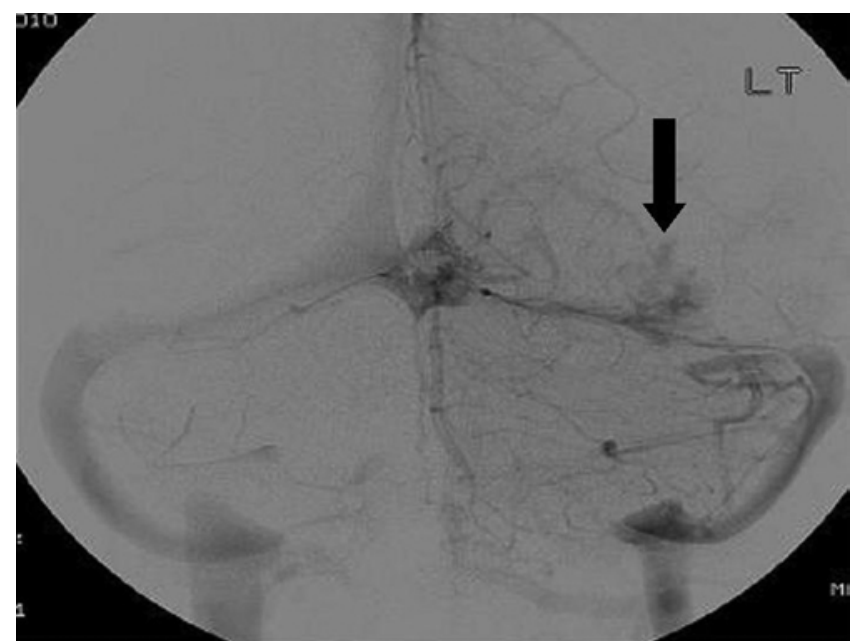

FIG. 2. Case 1. Anteroposterior left vertebral artery angiogram, capillary-venous phase, showing persistent blush (arrow) of a left tentorial lesion. No early draining vein or rapid transit of contrast was detected.

CT scan as the blood that jetted under the medial aspect of the mass and superiorly. There was no stump to coagulate but only a small hole in the MCA branch jetting arterial blood. This was covered with hemostatic agents and slight pressure was added with a cottonoid. After several minutes, there was no bleeding with the release of pressure. Despite preservation of the major sylvian vasculature, complete removal of the lesion, and evacuation of the hematoma, the brain swelling remained profound. The craniotomy was further enlarged, the dura opened more widely, the brain covered with a dural substitute, and the scalp closed without bone replacement. No blood transfusion was necessary.

\section{Postoperative Course}

After surgery, the patient had mild transient worsening of his central facial paresis, which has since completely resolved. Pathological features were diagnostic of capillary hemangioma. The diagnosis was corroborated by pathological review at another academic institution (Fig. 8). Cra-

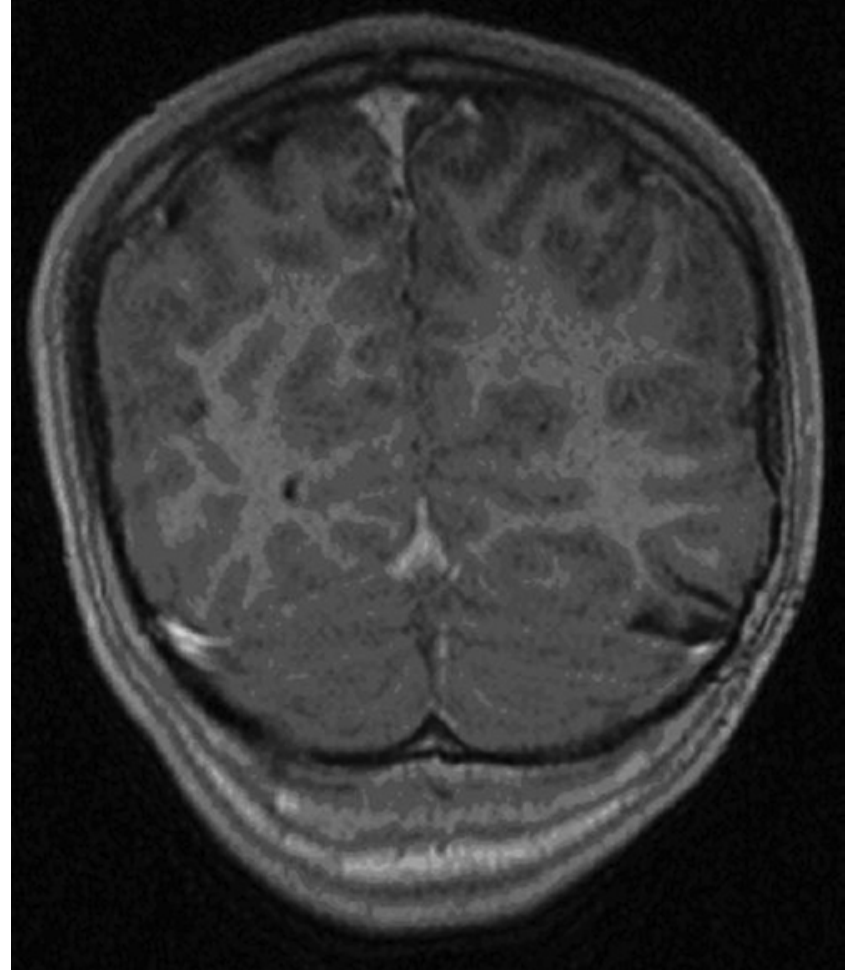

FIG. 4. Case 1. Coronal T1-weighted Gd-enhanced MR image 30 months postoperatively showing no evidence of residual or recurrent lesion, as well as resolution of edema.

nioplasty was scheduled for approximately 10 weeks after the initial craniotomy, but the cerebral edema was still too profound to place a prosthetic flap. Not until 6 months postoperatively did the cerebral edema clinically and radiologically diminish to allow for cranioplasty. Two years postoperatively he experienced a seizure after staying up all night. Academically he has performed no differently than prior to diagnosis. Immediate postoperative and longterm follow-up imaging showed no residual or recurrent mass but did display the brain swelling and encephalomalacia, respectively (Fig. 9).

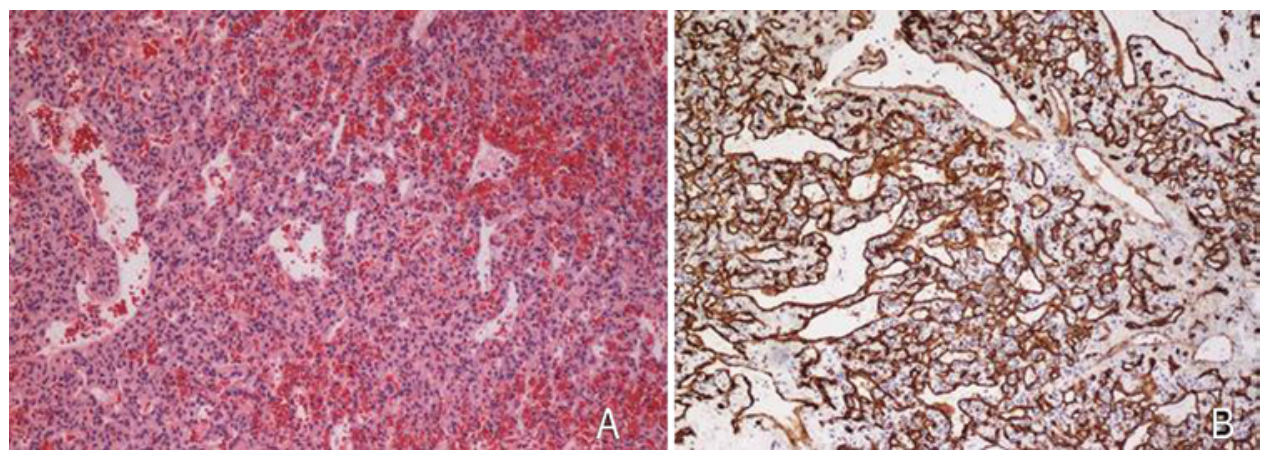

FIG. 3. Histopathological examination revealed a highly vascular lesion composed of variably sized vascular spaces. There were highly cellular areas composed of endothelial cells associated with small vascular spaces admixed with medium-sized, thin-walled vessels. Inhibin stain (not shown) was negative. Hemangioblastomas stain positive for inhibin. $\mathrm{H} \& \mathrm{E}$, original magnification $\times 100$ $(A)$; and CD-34 stain (B), original magnification $\times 100$. Figure is available in color online only. 

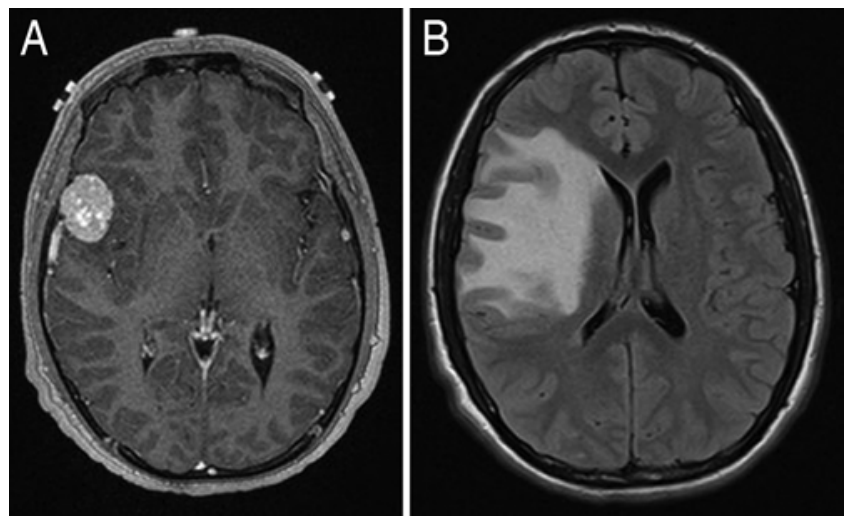

FIG. 5. Case 2. A: Axial T1-weighted Gd-enhanced MR image revealing a $28-\mathrm{mm}$, sharply circumscribed lesion in the right sylvian fissure. B: Axial FLAIR sequence showing extensive associated edema preoperatively.

\section{Discussion}

As reinforced by the 2 cases presented here, there is a predilection for more boys to be diagnosed with $\mathrm{ICH}$ in the pediatric age group. Including these 2 cases, the boy/ girl ratio is 11:5 for pediatric intracranial $\mathrm{ICH}$ (Table 1). When ICH presents in adulthood there is a predilection for women to be affected. In the 18 cases reviewed by Phi et al., of both children and adults with $\mathrm{ICH}$, males presented at a mean age of 4.8 years and females at a mean age of 22.5 years. ${ }^{13}$ While not all 14 patients reported on in the literature underwent a Gd-enhanced MRI, of those for whom the MRI findings were adequately described in the published reports, 9 had brightly enhancing lesions and 4 had significant perilesional edema. It is possible more than 9 and 4, respectively, had lesions with bright enhancement or significant perilesional edema, as in some reports either the representative images or their description was lacking.

The 2 patients described here had clinical presentations typical of raised intracranial pressure (ICP) due more to the profound edema than mass effect from the ICH itself. The cause of this edema is not clear. From this experience and the literature, ICHs associated with the tentorium or those found within the parenchyma tend to be associated with edema. Those ICHs that seemingly arise from the skull base or have wide dural bases mimicking meningiomas tend not to have associated edema (Table 1). It would be tempting to surmise that the lesion contains a high concentration of capillaries without a normal blood-brain barrier and this nest of leaky capillaries causes the edema. These lesions also may be associated with yet-to-be-defined abnormalities of the normal venous drainage, as seen with the coexistence of developmental venous malformations and cavernous malformations causing regional venous hypertension. This may explain why the second child required a prolonged time for edema resolution. Finally, as shown by Frei-Jones et al., these lesions can be associated with elevated vascular endothelial growth factor levels that may affect the capillary integrity of the blood-brain barrier within the surrounding brain. ${ }^{5}$

Additional causes of raised ICP other than edema in $\mathrm{ICH}$ have been reported as well. Brotchi et al. have de-

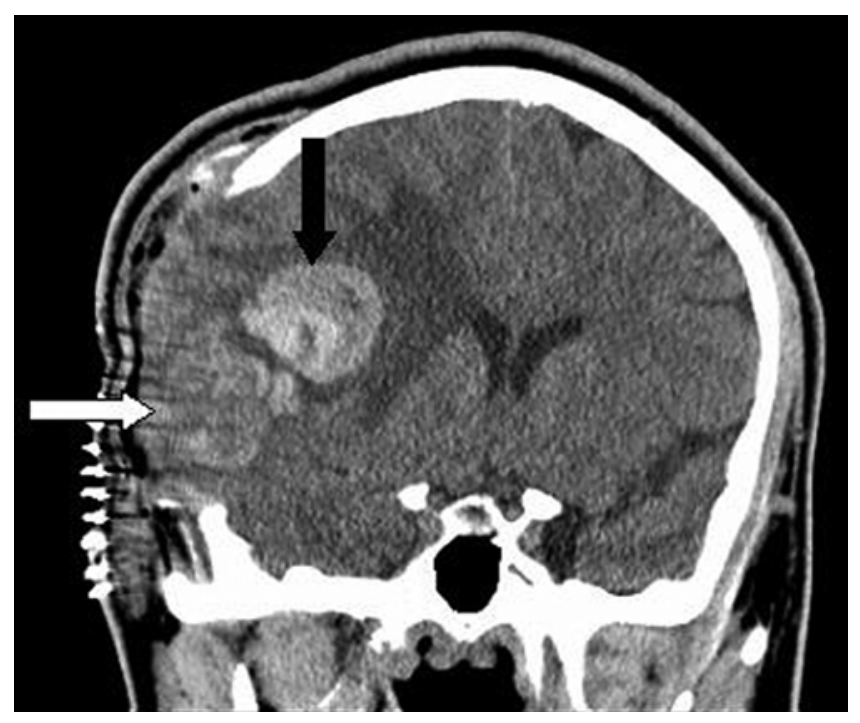

FIG. 6. Case 2. Coronal noncontrast cranial CT scan obtained after halting operation showing the unresected mass (white arrow) and new hematoma (black arrow) from spontaneous intraoperative bleeding.

scribed the case of a child presenting with elevated ICP secondary to an ICH that caused sagittal sinus obstruction. ${ }^{3}$ Other authors have reported children presenting with signs and symptoms of elevated ICP secondary to the shear size of the ICH itself., ${ }^{4,5,19}$ One infant died of a subarachnoid hemorrhage in association with a skull base ICH fed by the anterior choroidal artery. ${ }^{9}$

While few cases of ICH have been described, the MRI

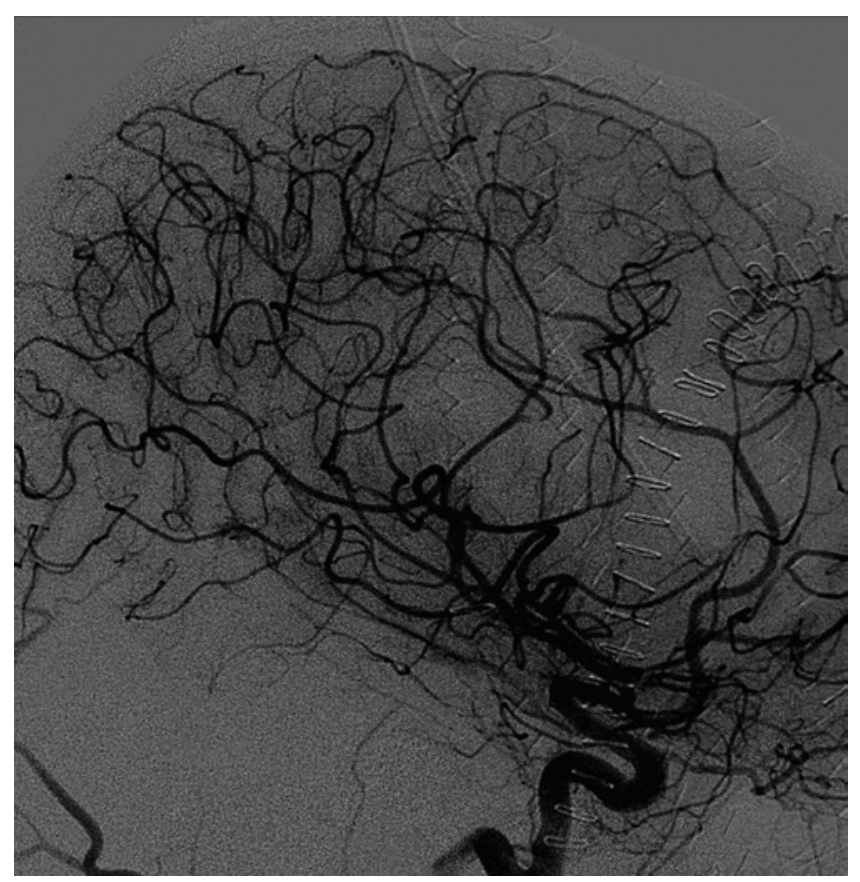

FIG. 7. Case 2. Lateral right internal carotid artery angiogram, late arterial phase, showing no draining vein or abnormal arterial flow. An external carotid artery angiogram (not shown) likewise showed no pathological findings. 


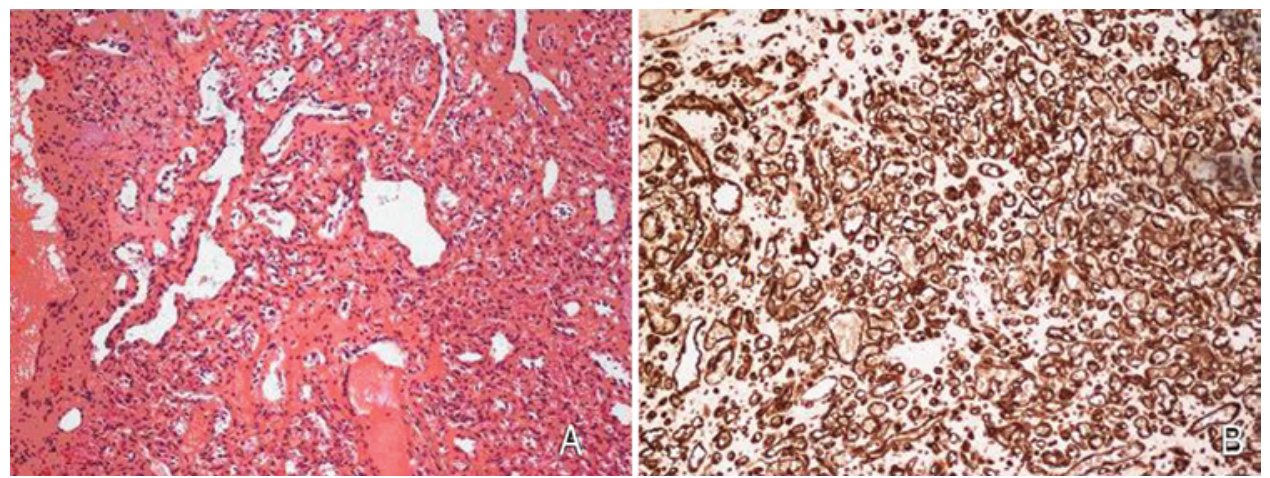

FIG. 8. Case 2. Histopathological examination revealed a highly vascular lesion composed of variably sized vascular spaces. There were highly cellular areas composed of endothelial cells associated with small vascular spaces admixed with medium-sized, thin-walled vessels. Inhibin stain (not shown) was negative. Hemangioblastomas stain positive for inhibin. $\mathrm{H}$ \& $\mathrm{E}$, original magnification $\times 100(A)$; and CD-34 stain (B), original magnification $\times 100$. Figure is available in color online only.

features at presentation in the 2 cases reported here were consistent with those in many prior reports of adults and children. ${ }^{14}$ A brightly enhancing, well-circumscribed lesion on T1-weighted images with Gd in close proximity to major vascular channels was seen in both cases. On T2-weighted or FLAIR images, signal change consistent with extensive perilesional edema was also present in both cases. Two reports have described lesions mimicking convexity meningiomas with broad-based dural attachments without cerebral edema. ${ }^{13,17}$ The case of a fourth ventricular ICH in a 3-month-old child presenting with hypotonia had no associated edema. ${ }^{8}$ Most reported cases of ICH in which angiography was performed preoperatively showed a significant blush of contrast filling in the capillary phase. Both pial and external carotid arterial supplies have been described. The angiographic findings seen in the 2 cases reported here, however, may have been distorted by the fact the angiograms were obtained after craniotomy, but before resection, and a degree of devascularization may have occurred. In the present 2 cases angiography showed either only a subtle blush in the capillary and venous phases or no detectable contrast filling. Some ICHs have been embolized preoperatively to assist with resection. ${ }^{4,11}$
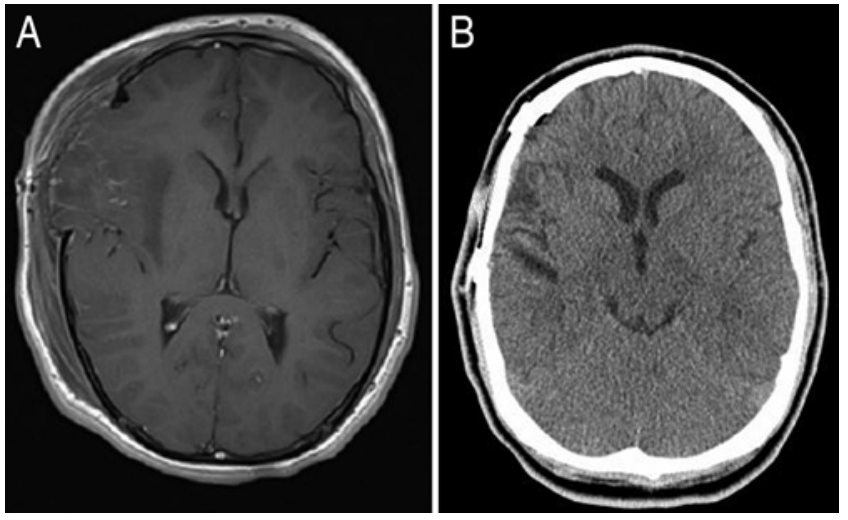

FIG. 9. Case 2. A: Axial T1- weighted Gd-enhanced MR image on postoperative Day 1 showing complete resection of the lesion but persistent edema and mushrooming of brain through craniotomy site. B: Noncontrast axial cranial CT scan 2 years after resection and subsequent cranioplasty showing resolution of edema and resultant encephalomalacia.
While also exceedingly rare, supratentorial hemangioblastomas would be considered in the differential diagnosis. The MRI findings of hemangioblastomas tend not to show the profound perilesional edema seen with ICHs, and vascular flow voids are often displayed. ${ }^{6}$ Association with cysts is common with hemangioblastomas, but not with ICHs, though 2 pediatric cases did have ICHs associated with cysts. ${ }^{13,16}$ The angiographic findings of hemangioblastomas tend to be more dramatic, with a very dense tumor blush, dilated feeding arteries, and dilated draining veins. In one child with an ICH, the angiographic findings shared similarities with those of hemangioblastomas. ${ }^{17}$ Papillary endothelial hyperplasia (PEH) is another vascular proliferative disorder to be considered in the differential diagnosis and is also exceedingly rare. PEH is thought to be an exuberant form of organizing thrombus that can arise in a preexisting vascular abnormality (intravascular) or hematoma (extravascular). ${ }^{12}$ MRI of PEH shows poor to variable enhancement, with evidence of old hemorrhage and variable amounts of mild edema. Histopathologically, numerous vascular channels surround fibrin cores. ${ }^{7}$ Evidence of preexisting hemorrhage and the histopathological characteristics of PEH were not seen in the 2 present cases.

Angiography in the setting of a possible ICH serves multiple purposes of excluding vascular anomalies such as arteriovenous malformation or aneurysm that may be treated by nonsurgical means, allowing for embolization in selected lesions, and providing the angioarchitecture of the lesion for the surgeon. Having angiographic information preoperatively in these 2 cases would have simplified the surgical management because the surgeon would have known that the lesions were not high-flow vascular lesions despite their intraoperative appearance.

Most reported cases have been treated with complete surgical resection. Because of the vascular nature of these lesions, either open or stereotactic biopsy would be ill advised were the lesion considered to be an ICH preoperatively. ${ }^{5}$ Some reports, however, have described partial resection of ICH in adults and children. These partially resected lesions often involved the skull base and cavernous sinus. The residual portion was then successfully treated with fractionated radiation. ${ }^{11,15}$

Two cases of patients with multiple ICHs have been 
treated successfully with resection of one lesion and medical management of the remaining lesions (steroids and alpha-interferon). The response of cutaneous capillary hemangiomas in infancy to exogenous steroids is a wellrecognized treatment to avoid surgery or to shrink lesions before undertaking surgery. ${ }^{10}$ Steroid receptor positivity was seen in 3 of 10 lesions involving the brain or spinal cord. ${ }^{2}$ Whether steroid receptor presence correlates with a therapeutic response to glucocorticoids is not known. One infant with a giant lesion that could not be safely resected was successfully managed by undergoing a 19-month course of thalidomide, an antiangiogenic agent, which shrunk the lesion to a small residual mass. Treatment response correlated with systemic vascular endothelial growth factor levels. ${ }^{5}$ Similarly using another antiangiogenic agent, bevacizumab, along with temozolomide, an intracranial cellular hemangioma in an infant was successfully treated. ${ }^{18}$ Cellular hemangioma is considered a more aggressive lesion but is related to ICH along the spectrum of vasoproliferative lesions.

As for the 2 cases in the present report, the lessons learned are several. While the preoperative imaging was "typical" for ICHs, these lesions are exceedingly rare and an ICH was not considered preoperatively. Given the vivid enhancement with gadolinium and the lesion's proximity to major vascular structures, some type of angiographic imaging would have been helpful preoperatively to better understand the vascular nature of the lesion in each case and provide confidence that a high-flow vascular lesion was not present. Formal angiography has been used successfully in reported cases more than MR angiography. Angiography also allows for embolization if indicated. There have been no reported cases in which CT angiography was performed.

Preoperative consideration of ICHs in the differential diagnosis could have allowed for preoperative medical management with steroids or other agents discussed above to possibly reduce the profound cerebral edema associated with these lesions and thus make the operation easier and with less morbidity. The exact medical regimen, however, would have been empirical and not necessarily successful.

Also given the edema present with these relatively small lesions, a wide surgical field should be draped. While neurosurgeons may take pride in removing intracranial lesions through the smallest of craniotomies, with a potential ICH the surgeon should be prepared to undertake a large decompressive craniotomy if intraoperative brain swelling otherwise cannot be controlled.

While ICHs seem not to present because of hemorrhage, intraoperative spontaneous or surgeon-induced bleeding (e.g., from a biopsy) can be an intraoperative complication requiring an array of hemostatic agents and need for volume, blood, and coagulation factor replacement. Hence, the surgical and anesthetic teams should be prepared beyond the normal preparation for a routine pediatric brain tumor case.

\section{Conclusions}

ICHs are benign vascular lesions that can be surgically treacherous because of their vascularity and associated brain edema. A brightly enhancing, well-circumscribed mass shown on MRI near the dural sinuses or major vascular structures, especially with significant perilesional edema, should alert the surgeon to the possibility of an ICH. Appropriate imaging studies to exclude high-flow vascular lesions preoperatively are indicated if an ICH is in the differential diagnosis. If the diagnosis is known through biopsy, incomplete resection, or complete resection of one of multiple ICHs, then medical and radiotherapy options seem effective for residual lesions. Preoperative medical regimens to reduce brain swelling should be considered. The surgeon and anesthetic team should be prepared for spontaneous hemorrhage, substantial blood loss, and difficult-to-control brain swelling. Surgical excision is curative.

\section{Acknowledgments}

I thank radiologist Dr. Warren Goldstein for performing the emergency angiography, pathologist Dr. Richard Halbert for obtaining photomicrographs of the resected lesions, and pathologist Dr. Peter Burger for reviewing the specimens.

\section{References}

1. Abe M, Misago N, Tanaka S, Masuoka J, Tabuchi K: Capillary hemangioma of the central nervous system: a comparative study with lobular capillary hemangioma of the skin. Acta Neuropathol 109:151-158, 2005

2. Abe M, Tabuchi K, Tanaka S, Hodozuka A, Kunishio K, Kubo N, et al: Capillary hemangioma of the central nervous system. J Neurosurg 101:73-81, 2004

3. Brotchi J, Baleriaux D, Kalangu KKN, Morelli D, Rodesch $\mathrm{G}$, Rorive S, et al: Capillary hemangioma in the superior sagittal sinus as a rare cause of intracranial hypertension in a child: case report. Neurosurgery 57:E815-E816, 2005

4. Daenekindt T, Weyns F, Kho KH, Peuskens D, Engelborghs $\mathrm{K}$, Wuyts J: Giant intracranial capillary hemangioma associated with enlarged head circumference in a newborn. J Neurosurg Pediatr 1:488-492, 2008

5. Frei-Jones M, McKinstry RC, Perry A, Leonard JR, Park TS, Rubin JB: Use of thalidomide to diminish growth velocity in a life-threatening congenital intracranial hemangioma. J Neurosurg Pediatr 2:125-129, 2008

6. Ho VB, Smirniotopoulos JG, Murphy FM, Rushing EJ: Radiologic-pathologic correlation: hemangioblastoma. AJNR Am J Neuroradiol 13:1343-1352, 1992

7. Karamchandani J, Vogel H, Fischbein N, Gibbs I, Edwards MSB, Griffith H IV: Extravascular papillary endothelial hyperplasia mimicking neoplasm after radiosurgery: case report. Neurosurgery 70:E1043-E1048, 2012

8. Karikari IO, Selznick LA, Cummings TJ, George TM: Capillary hemangioma of the fourth ventricle in an infant. Case report and review of the literature. J Neurosurg 104 (3 Suppl):188-191, 2006

9. Le Bihannic A, Michot C, Heckly A, Loget P, Beucher A, Brassier $\mathrm{G}$, et al: Capillary haemangioma arising from the anterior choroidal artery. Childs Nerv Syst 21:265-271, 2005

10. Mirza B, Shi WY, Phadke R, Holton JL, Turner C, Plant GT, et al: Strawberries on the brain-intracranial capillary hemangioma: two case reports and systematic literature review in children and adults. World Neurosurg 80:900.e13-900.e1, 2013

11. Morace R, Maronggiu A, Vangelista T, Galasso V, Colonnese $\mathrm{C}$, Giangaspero F, et al: Intracranial capillary hemangioma: a 
description of four cases. World Neurosurg 78:191.e15-191. e21, 2012

12. Park KK, Won YS, Yang JY, Choi CS, Han KY: Intravascular papillary endothelial hyperplasia (Masson tumor) of the skull: case report and literature review. J Korean Neurosurg Soc 52:52-54, 2012

13. Phi JH, Kim SK, Cho A, Kim DG, Paek SH, Park SH, et al: Intracranial capillary hemangioma: extra-axial tumorous lesions closely mimicking meningioma. J Neurooncol 109:177-185, 2012

14. Simon SL, Moonis G, Judkins AR, Scobie J, Burnett MG, Riina HA, et al: Intracranial capillary hemangioma: case report and review of the literature. Surg Neurol 64:154-159, 2005

15. Tsao MN, Schwartz ML, Bernstein M, Halliday WC, Lightstone AW, Hamilton MG, et al: Capillary hemangioma of the cavernous sinus. Report of two cases. J Neurosurg 98:169174,2003

16. Uyama A, Kawamura A, Akiyama H, Nakamizo S, Yamamoto K, Nagashima T, et al: A case of cerebellar capillary hemangioma with multiple cysts. Pediatr Neurosurg 44:344-349, 2008

17. Willing SJ, Faye-Petersen O, Aronin P, Faith S: Radiologicpathologic correlation. Capillary hemangioma of the meninges. AJNR Am J Neuroradiol 14:529-536, 1993
18. Yeo KK, Puscasiu E, Keating RF, Rood BR: Durable response of intracranial cellular hemangioma to bevacizumab and temozolomide. J Neurosurg Pediatr 11:682-686, 2013

19. Zheng SP, Ju Y, You C: Giant intracranial capillary hemangioma in a 3-year-old child: case report and literature review. Clin Neurol Neurosurg 114:1270-1273, 2012

\section{Disclosures}

The author reports no conflict of interest concerning the materials or methods used in this study or the findings specified in this paper.

\section{Supplemental Information}

Previous Presentations

Presented at the 41st Annual Meeting of the AANS/CNS

Section on Pediatric Neurological Surgery, St. Louis, Missouri, November 27-30, 2012.

\section{Correspondence}

Paul A. Grabb, 2401 Gillham Rd., Kansas City, MO 64108. email: pagrabb@cmh.edu. 